\title{
DYNAMICS OF FLAME PROPAGATION FROM LOCALIZED IGNITION IN RICH HYDROGEN/AIR MIXTURES: EFFECTS OF ELEVATED PRESSURE AND TEMPERATURE
}

\author{
STEPHEN D. TSE* \\ HONG ZHAO \\ Department of Mechanical and Aerospace Engineering, \\ Rutgers University, Piscataway, New Jersey, USA
}

\begin{abstract}
A computational study was conducted on expanding spherical premixed flames to investigate the dynamics of the stretch-affected, onedimensional, pulsating instability at elevated pressures and temperatures. Rich $\mathrm{H}_{2}$ /air mixtures were investigated using a time-dependent, spherically symmetric code with detailed chemistry, transport, and radiation submodels. The study shows that the region of instability in the pressure versus equivalence ratio domain for outwardly propagating flames is significantly narrowed when compared with that for planar flames due to the presence of the local ignition limit despite stretchinduced pulsation modifying the other boundary. Moreover, a region of one oscillation ending in extinction (even under adiabatic conditions which are not present in planar flames) has been discovered. However, similar to planar flames, radiative loss in outwardly propagating spherical flames reduces the region of instability due to transient extinction. Interestingly, this limit lies next to and just below the concentration limit for ignition. For the same ignition/extinction reasons, the region of instability is expanded at elevated temperatures due to increased flammability. The calculations show that the dynamics of spherically expanding flames is significantly different than that for planar flames.
\end{abstract}

Received 14 April 2004; accepted 14 September 2004.

This work was partially supported by the Zonta International Amelia Earhart Fellowship and the National Science Foundation. Special thanks are due to Mr. Sravan Ravinutala for his help with the numerical code.

*Address correspondence to sdytse@rci.rutgers.edu 
As a result, the near-limit phenomenon is extremely geometry dependent, thereby having an impact on practical considerations of flammability and stability.

Keywords: spherical flames, instability, flame propagation, flammability limits, ignition

\section{INTRODUCTION}

The propagation of laminar premixed flames in mixtures with sufficiently greater than unity Lewis numbers $(L e)$ is susceptible to diffusionalthermal pulsating instability, which has been shown analytically (Joulin and Clavin, 1979; Sivashinsky, 1974, 1977) and numerically (Rogg, 1982) with one-step chemistry. Numerical studies with detailed chemistry (Christiansen et al., 1998, 2001; Goyal et al., 1995; He and Clavin, 1993; Kailasanath et al., 1993), for hydrogen/air or hydrogen/oxygen flames approaching the rich limit where $L e \gg 1$, have also been conducted. In consideration of detailed chemistry and transport, Christiansen et al. (1998) showed that the transition boundary from steady to pulsating propagation can be adequately described by Sivashinsky's (1977) criterion:

$$
Z e(L e-1)>4(1+\sqrt{3}) \approx 11
$$

using appropriately extracted $L e$ and Zeldovich numbers $(Z e)$ characterizing steady, one-dimensional, adiabatic flame propagation in the doubly infinite domain. As such, pulsation is promoted for large values of $L e$ and $Z e$. Additionally, the presence of heat loss has also been examined. Joulin and Clavin (1979), using a linear heat-loss model, found that the critical $L e$ beyond which the flame becomes unstable decreases in the presence of heat loss. However, Christiansen et al. (1998) found that, when radiative heat loss is considered, the limit for the onset of oscillatory propagation appears to be minimally affected but is important when the oscillations are strong enough for the heat loss to extinguish the flame during the weakly burning portion of its cycle. The rich flammability limit determined in this manner occurs at a lower equivalence ratio than the steady flame turning point.

Note that the aforementioned works have examined the pulsating modes of flame propagation for the one-dimensional planar flame; Goyal et al. (1995) also examined the curved flame in a burner-stabilized configuration, but not the freely propagating spherical flame. Nonetheless, 
the onset of the instability is expected to be altered in the presence of aerodynamic stretch. For example, Farmer and Ronney's (1990) numerical work on outwardly propagating flames with one-step chemistry indicated that oscillation is promoted at small radii (i.e., strong positive stretch) and is damped at large radii. More recently, Sung et al. (2002), in their asymptotic analyses and computational treatments of the negatively stretched, inwardly propagating spherical flame and the positively stretched counterflow flame in rich hydrogen/air mixtures, showed that positive stretch promotes the onset of flame pulsation whereas negative stretch retards it.

The present study is motivated by several considerations. First, the near-limit phenomenon is expected to be extremely geometry dependent, thereby having an impact on our understanding of various flame phenomena based on the concept of steady flame propagation. There has been debate regarding whether the actual flammability limit should be based on the idealized planar flame configuration or the spherical flame configuration. The fundamental flammability limit, which is traditionally defined (Law and Egolfopoulos, 1992; Spalding, 1957; Williams, 1985) by the inability of the one-dimensional planar flame to propagate steadily through the doubly infinite domain with radiative heat loss, is contrasted by practical considerations of initially establishing a propagating flame through a localized ignition source of finite energy. Planar flames that can exist in the doubly infinite domain for mixture compositions below this (e.g., rich) fundamental flammability limit may not be dynamically attainable from initially localized flame structures. The concept of the existence of one-dimensional planar flames which may not be dynamically realized directly affects the stability and ignition boundaries of reactive mixtures containing the pulsating regime. This is examined with and without radiative loss for elevated pressures and temperatures as a function of equivalence ratio. Second, with the dynamics of spherically expanding flames significantly different than that for planar flames, the influence of positive stretch on the onset instability boundaries, as well as on flame response throughout the propagation expanding process, is assessed. Third, the influence of the pulsating instability on outwardly propagating flame extinction boundaries is examined. Since the spherical flame is subjected to overall decreasing magnitudes of positive stretch as it propagates outward, the overall maximum temperature increases for the $L e>1$ flame, burning stronger as it expands to that of the planar flame. Thus, the relevant limit 
phenomenon for nonpulsating, $L e>1$ flames is not extinction but rather ignition, because once the flame is established, it has already survived the most severe stretch situation. However, such an analysis does not consider the regime of the pulsating instability, which comes into further prominence due to its "enhancement" by positive stretch. As a result, transient extinction can occur even after ignition due to such oscillatory flame response. Fourth, because the pulsation behavior of the spherical flame itself affects the instantaneous stretch $\kappa=2 / r_{f}\left(d r_{f} / d t\right)$, in contrast to the monotonically decreasing positive stretch experienced by the stable flame, it is interesting to assess whether and how the pulsating flame responds to its own perturbations. Finally, we note that the numerical study of Farmer and Ronney (1990) was conducted for simplified chemistry and transport and that the focus of the study was not on the effects of stretch on flame pulsation. As such, we computationally simulate the outwardly propagating flame with detailed chemistry and transport to definitively establish the physical nature of such flames in the presence of naturally changing positive stretch. Such a study has direct impacts on applications involving such flame geometries as well as with all practical flames experiencing flow nonuniformity, flame curvature, and/or flame unsteadiness.

To explore such inherently unsteady and history-sensitive flame responses at elevated pressures and temperatures, a detailed unsteady computational simulation using realistic descriptions of chemical, diffusive, and radiative aspects of the highly nonequidiffusive, $L e>1$, rich hydrogen/air system has been conducted. The problem studied is defined in the next section, which is followed by presentation and discussion of results.

\section{PROBLEM DEFINITION}

The simulation employs the one-dimensional, finite-difference, implicit time-dependent flame code of Rogg and Wang (1995), with adaptive nonuniform gridding, using detailed chemical, transport, and optically thin radiation models. Because chemistry is not the main focus of the present work, only a single detailed kinetic mechanism (Meuller et al., 1999), involving 9 species and 21 elementary reactions, is applied here. Nonetheless, chemistry influences the problem by changing $Z e$ for different pressures, which has been discussed by Christensen et al. (1998, 2001). Use of a detailed chemical mechanism captures the pressure 
dependence of the overall activation energy, inherently applies the "true" Lewis number, and accurately resolves the concentrations of product species involved in radiative processes. Our results confirmed that the phenomenon examined is due to the thermal-diffusive pulsating instability and not to chemical oscillations.

Using the small-Mach-number approximation, where the thermodynamic pressure is constant throughout the flow field, the governing equations are

$$
\begin{gathered}
\frac{\partial \rho}{\partial t}+u \frac{\partial \rho}{\partial r}=-\frac{\rho}{r^{2}} \frac{\partial\left(r^{2} u\right)}{\partial r} \\
c_{p} \rho\left(\frac{\partial T}{\partial t}+u \frac{\partial T}{\partial r}\right)=\frac{1}{r^{2}} \frac{\partial}{\partial \boldsymbol{r}}\left(r^{2} \lambda \frac{\partial T}{\partial \boldsymbol{r}}\right)-\sum_{i=1}^{N} h_{i} \omega_{i}-\frac{\partial T}{\partial r} \sum_{i=1}^{N} c_{p_{i}} j_{i}-q_{\mathrm{rad}}+\boldsymbol{q}_{e} \\
\rho\left(\frac{\partial Y_{i}}{\partial \boldsymbol{t}}+u \frac{\partial Y_{i}}{\partial \boldsymbol{r}}\right)=-\frac{1}{r^{2}} \frac{\partial\left(r^{2} j_{i}\right)}{\partial \boldsymbol{r}}+\omega_{i} \\
p=\frac{\rho R T}{M}
\end{gathered}
$$

where $t$ is the time; $r$ is the radius; $u$ is the radial velocity; $\rho$ is the density; $T$ is the temperature; $c_{p}$ and $\lambda$ are the constant-pressure heat capacity and thermal conductivity of the mixture, respectively; $p$ is the pressure; $R$ is the universal gas constant; $M$ is the mass-averaged molecular weight of the mixture; and $N$ is the total number of species. Furthermore, $Y_{i}, \omega_{i}$, $h_{i}$, and $j_{i}$ are the mass fraction, mass rate of production, specific enthalpy, and mass diffusion flux of the $i$ th species, respectively.

For optically thin radiation,

$$
q_{\mathrm{rad}}=4 \alpha_{p} \sigma\left(T^{4}-T_{\infty}^{4}\right)
$$

where $\sigma$ is the Stefan-Boltzmann constant and $\alpha_{p}$ the total Planck mean absorption coefficient. Radiation heat loss is considered to be emitted only from $\mathrm{H}_{2} \mathrm{O}$. Planck mean absorption/emission coefficients based on a wideband model are taken from Hubbard and Tien (1978). 
Ignition is achieved by energy deposition centered at zero radius such that

$$
\begin{aligned}
& q_{e}=\Delta \exp \left[-\left(\frac{r}{r_{s}}\right)^{8}\right] \quad t \leq \tau_{s} \\
& q_{e}=0 \quad t>\tau_{s}
\end{aligned}
$$

where $\Delta$ is the density, $r_{s}$ is the radius, and $\tau_{s}$ is the duration of the source energy. Typical ignition energy parameters used are $\Delta=$ $2 \times 10^{10}\left(\mathrm{~W} / \mathrm{m}^{3}\right) \times \mathrm{p}(\mathrm{atm}), \mathrm{r}_{\mathrm{s}}=2 \mathrm{~mm}$, and $\tau_{\mathrm{s}}=50 \mu \mathrm{s}$, which roughly corresponds to $35 \times p(\mathrm{~atm}) \mathrm{mJ}$ of total energy. He and Clavin (1993) found that the critical size of a hot pocket for initiating a deflagration wave is a function of the equivalence ratio: as the hydrogen-rich flammability is approached, the critical radius becomes much larger than the flame thickness and, moreover, diverges before reaching the planar flame limit. Because the main focus of the present study is not ignition, the size of the ignition kernel and ignition time duration were fixed so that the flame responses could be compared on a relatively equal basis. Different localized ignition kernels of reasonable temporal and spatial sizes were tested for the examined pressure range, and the ignition energy parameters given earlier resulted in flame responses that are independent of the ignition source, after a rapid transient period. It is conceivable that large ignition kernels extend the flammability limit boundary, but only moderately, with hard limits as found in the literature ( $\mathrm{He}$ and Clavin, 1993). Nonetheless, all of our ignition energies are at least an order of magnitude larger than the minimum ignition energy. Otherwise the results confirm that the phenomenon is controlled by the dynamics of the flame front instead of the ignition energy, although ignition is the subject of future study. We track the flame front by defining the flame radius as the location of peak heat release.

At $r=0$, zero-gradient conditions are enforced. At the ambient side, boundary conditions imposed are temperature $T_{\infty}$, fixed mixture composition, and radiative emissivity of unity. An outer boundary radius of $1 \mathrm{~m}$ is used. System pressures range from 1 to $20 \mathrm{~atm}$, and ambient temperatures range from 300 to $500 \mathrm{~K}$. Verification tests, with fixed grid points and smaller time steps, showed that the obtained results (including those for the pulsating flames) were independent of the autogrid schemes in the code and the time step used. 


\section{RESULTS AND DISCUSSION}

\section{Pressure Effects and Flammability Boundaries}

This work was partially motivated by the fact that the diffusive-thermal oscillatory behavior as predicted for rich hydrogen/air mixtures has not yet been observed in experiments. Figure $1 a$ (from Christiansen et al., 2001) shows the stability and extinction boundaries of rich hydrogen/ air flames from 1 to $20 \mathrm{~atm}$ of the one-dimensional planar flame in the doubly infinite domain. Radiative loss is the mechanism responsible
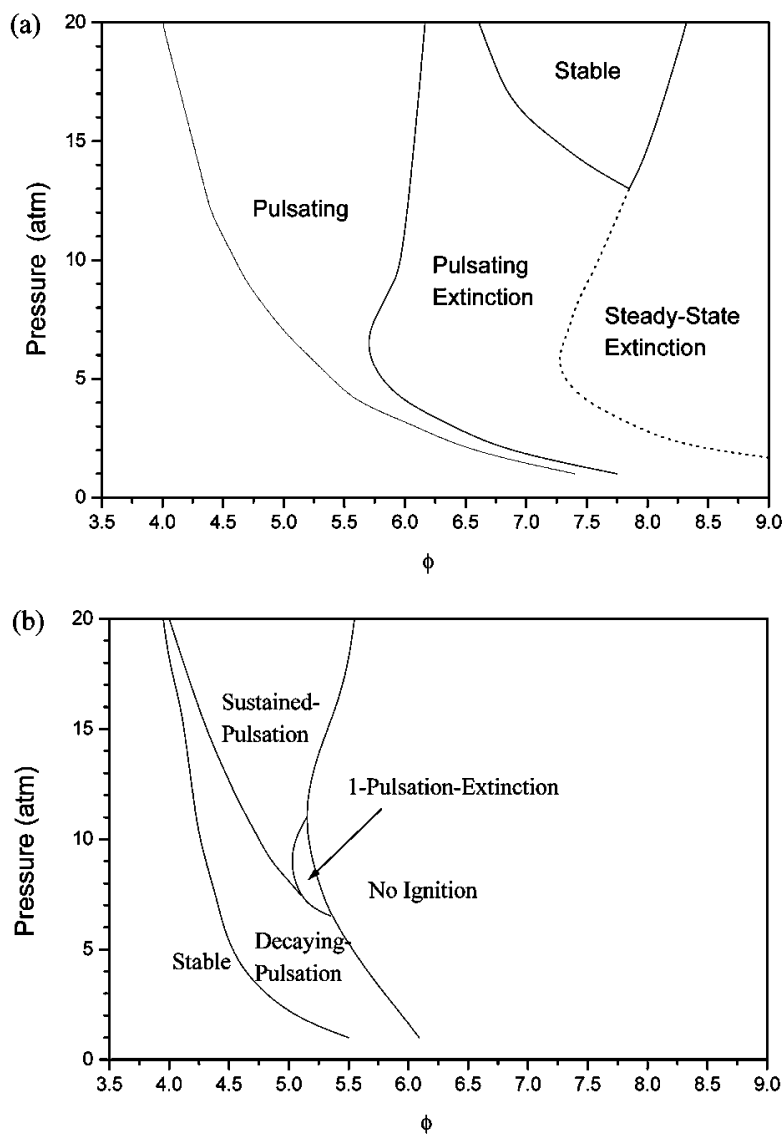

Figure 1. Stability and extinction boundaries (a) for planar flames of rich $\mathrm{H}_{2} /$ air (Christiansen et al., 2001) and (b) for adiabatic, centrally ignited, spherically propagating rich $\mathrm{H}_{2}$ /air spherical flames from 1 to $20 \mathrm{~atm}$ at $298 \mathrm{~K}$ ambient. 
for the pulsating extinction regime of the map, where the oscillatory nature of the instability facilitates flame extinction during its weakly burning stage. As can be seen, the pulsating instability tends to reduce the rich fundamental flammability limit (steady-state extinction). From 1 to $5 \mathrm{~atm}$, the onset of the pulsating regime ranges from about $\phi=7.4$ to 5.4 , respectively. However, these mixtures may not be ignitable by spherical hot pockets or localized energy sources. Figure $1 b$ shows the stability, extinction, and nonignition boundaries of adiabatic, spherically propagating, rich hydrogen/air flames established by local ignition from 1 to $20 \mathrm{~atm}$ at $298 \mathrm{~K}$ ambient. It is revealed that, from 1 to $5 \mathrm{~atm}$, flames cannot be ignited from about $\phi=6.1$ to 5.4 , respectively. As mentioned in the foregoing discussions, these mixtures are not ignitable even under adiabatic conditions, identifying a rich flammability limit of the mixture for situations involving ignition kernels. Although the ignition limit may be extended to larger equivalence ratios by employing large ignition kernels on the order of several centimeters in radius (He and Clavin, 1993), a diverging size limit still exists for critical equivalence ratios (He and Clavin, 1993), as a function of pressure, that have yet to be established in the literature for hydrogen/air flames. The determination of this limit is parametrically intensive and beyond the scope of the present study. For our cases, doubling the size of the kernels showed no difference in the boundary to within $\Delta \phi=0.05$. Nonetheless, even by employing large ignition kernels, which may not be practically feasible, the pulsating phenomenon is still not likely to be experimentally observed for this pressure range due to the critical limit. At higher pressures, with the flame thickness significantly reduced, larger ignition kernels are expected to minimally affect the ignition limit found in the present work; moreover, their use would obscure the study of the flame responses at high stretch rates (i.e., small radii).

With further consideration of this "adiabatic" no-ignition boundary (Figure $1 b$ ), not only is the pulsating regime of Figure $1 a$ imposed upon, but the radiatively induced pulsating extinction limit of Figure $1 a$ is precluded. The second stable regime at high pressures (Figure $1 a$ ) is also excluded, thereby establishing a rich flammability limit significantly far from the fundamental static limit. Nonetheless, with the onset of instability shifting to leaner equivalence ratios with increasing pressure (Figure 1a), a widening regime of sustained pulsating propagation for locally ignited spherical flames is established at pressures greater than 6 atm. Figure 2 shows a characteristic high-pressure spherically 


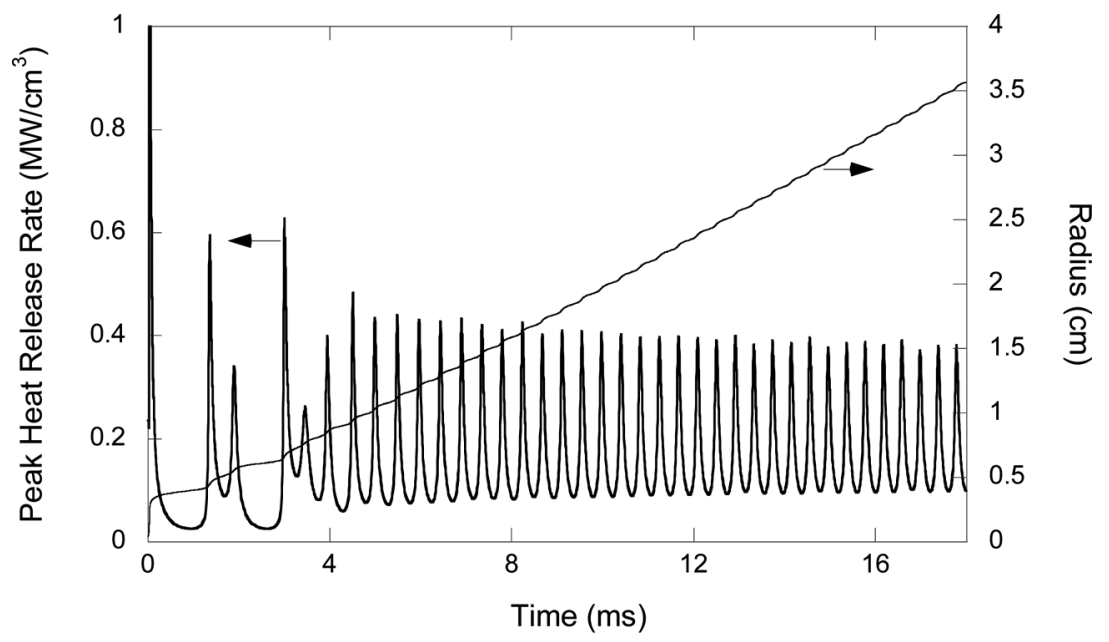

Figure 2. Adiabatic, outwardly, spherically propagating flame leading to sustained monochromatic oscillation at $11 \mathrm{~atm}, \phi=4.7$.

propagating flame of eventual sustained monochromatic oscillation that may be experimentally observed. Analyses of the oscillations within this regime show a slight increase in frequency with increasing flame radius (decreasing positive stretch). The calculations also confirm that pressure and equivalence ratio affect the oscillation frequency, as found by Christiansen et al. (2001) using Sivashinsky's (1977) prediction that the period of oscillation $\left(\tau_{p}\right)$ at the onset of instability scales linearly with the flame time $\left(\tau_{f}\right)$. With the characteristic flame time defined as $\tau_{f}=\lambda \rho /\left(\boldsymbol{m}^{0}\right)^{2} c_{p}$, one can see that, for a given equivalence ratio, the period of oscillation will decrease for higher pressures, due to larger mass burning rates. For a given pressure, the period of oscillation will increase for larger equivalence ratios, due to smaller mass burning rates.

The left boundary of the sustained pulsation regime of Figure $1 b$ corresponds to that for the planar pulsating regime of Figure $1 a$. Due to positive-stretch-induced instability, a decaying pulsation regime is found to the left of the sustained pulsating regime; Figure 3 shows a characteristic case. A criterion of at least one period of oscillation, with amplitude of at least $1 \%$ in the peak heat release, is used to demark this regime from the further-left-lying stable flame regime to within $\phi=0.1$. Although the decaying oscillation regime appears quite large below $5 \mathrm{~atm}$, it is mainly composed of one-period oscillations of small 


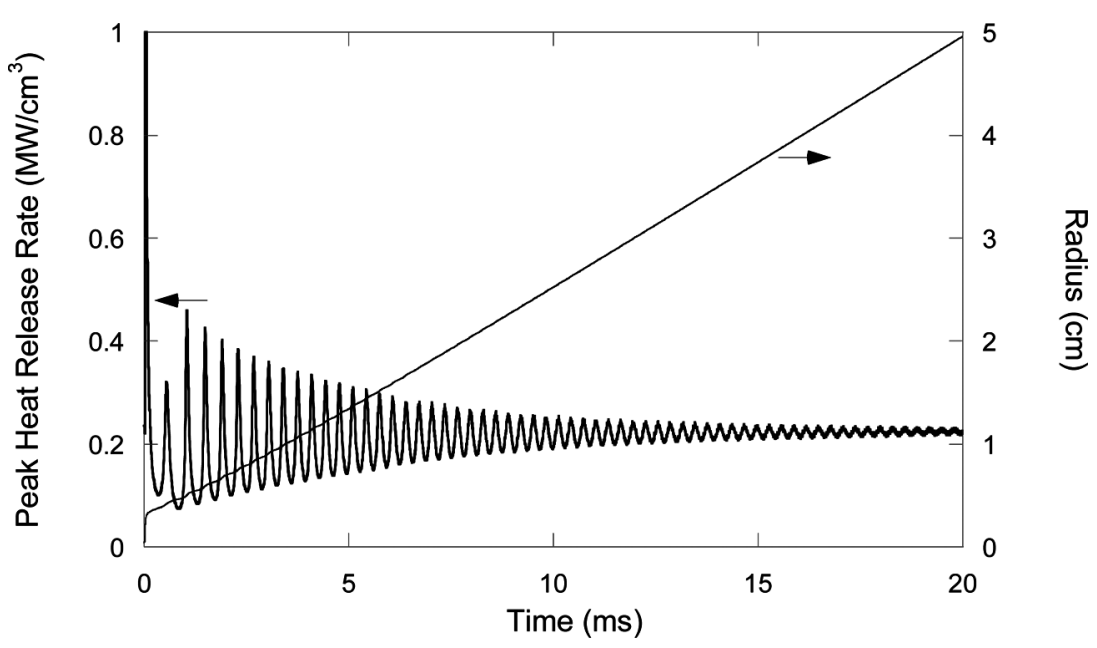

Figure 3. Adiabatic, outwardly, spherically propagating flame of decaying oscillation at $11 \mathrm{~atm}, \phi=4.6$.

amplitude, which are not likely to be observed experimentally. Moreover, note that, even for the case presented in Figure 3, oscillations in the flame-front position would be very difficult to observe experimentally. Because the magnitude of oscillation in the flame movement is small, tracking peak heat release through $\mathrm{OH}$ chemiluminescence using a fast-responding, filtered radiometer or photomultiplier would be the best experimental diagnostic. With the experimental setup of Tse et al. (2000), it may be possible to observe these hydrogen/air outwardly propagating flames at such pressures and equivalence ratios. Nevertheless, despite the presence of positive-stretch-induced pulsation, the overall window of pulsating behavior is significantly constricted by the ignition limit.

Figure $1 b$ displays an "island" regime (labeled 1-pulsation-extinction), adjacent to the ignition limit, of a single heat-release "burst" after ignition, with subsequent extinction; Figure 4 shows a characteristic case. Such an adiabatic mode of extinction is not available to the planar flame. Explanation of this phenomenon requires some discussion of stretch effects on the pulsating instability.

Figure 5 shows a case where the spectrum of the various modes of pulsation is stratified by the "average" stretch experienced by the outwardly propagating flame. At small radii (i.e., high stretch), the bursting or hibernation mode is manifested; at moderate radii (i.e., moderate 


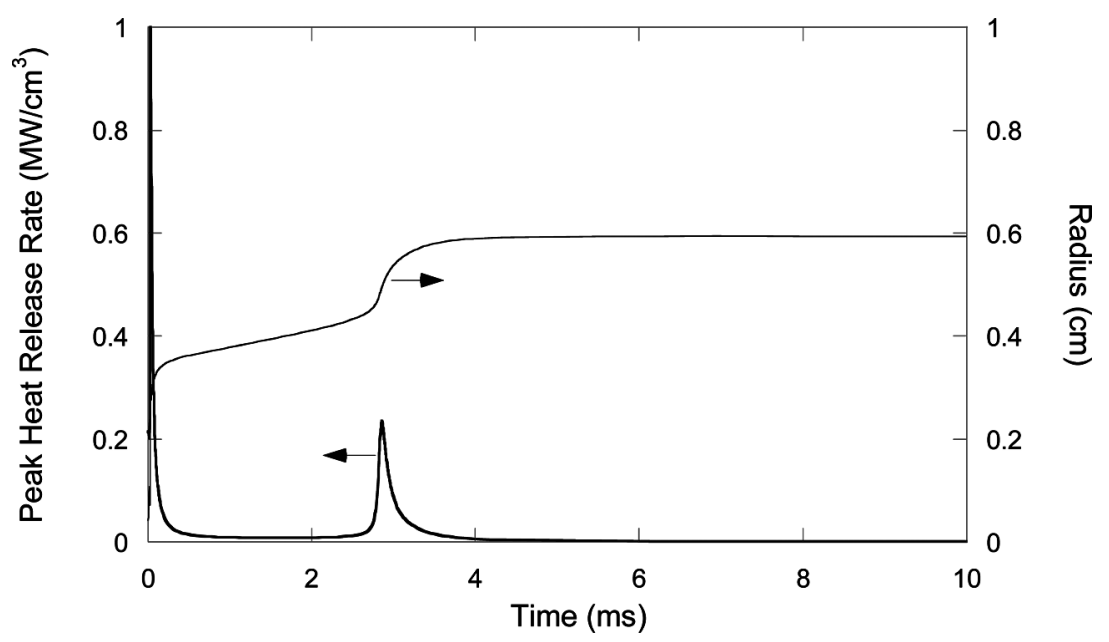

Figure 4. Adiabatic, outwardly, spherically propagating flame of single heat-release burst then extinction at $8 \mathrm{~atm}, \phi=5.15$.

stretch), the period doubling mode is encountered; and at large radii (i.e., low stretch), the monochromatic mode is sustained. The flame response to decreasing magnitudes of positive stretch is analogous to

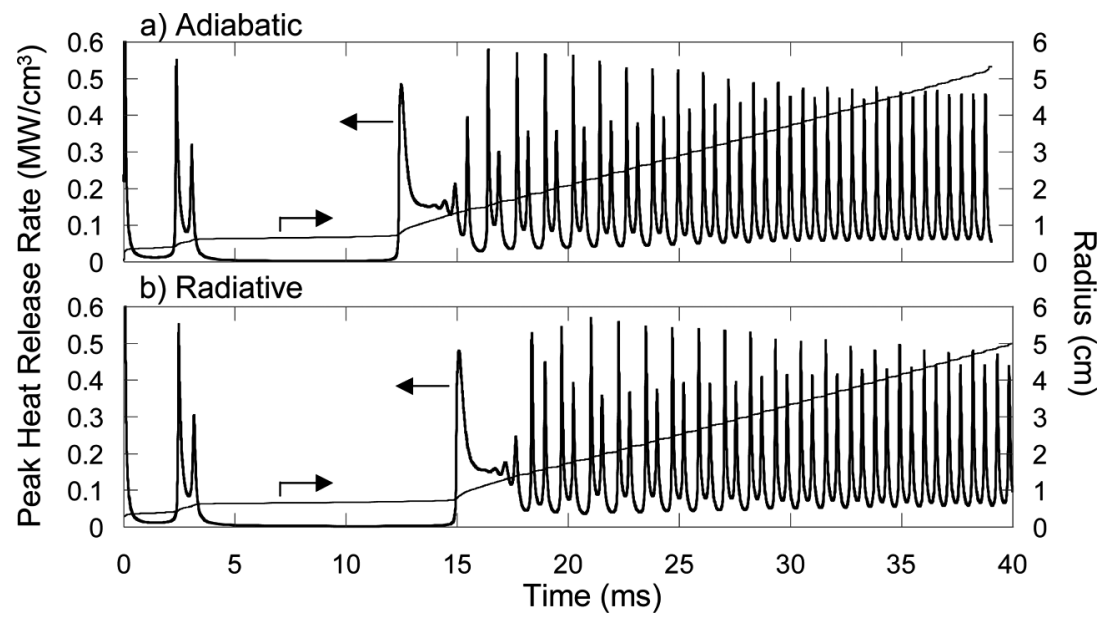

Figure 5. Outwardly, spherically propagating flame of sustained pulsation at $11 \mathrm{~atm}$, $\phi=4.8$. 
the response of adiabatic freely propagating planar hydrogen/air flames in the pulsating regime with increasing equivalence ratio. These results are consistent with those of Sung et al. (2002) for counterflow flames subjected to progressively increasing strain rate; this point will be discussed in more detail later. The flame of Figure 5 is sufficiently strong enough to survive the hibernation case and then continue to propagate as a planar pulsating flame, even with radiative loss. Note that the radiative case, when compared with the adiabatic case, extends the duration of diminished reactivity, as expected, but does little to affect the flame behavior once regular pulsations resume. However, for relatively weaker, or richer, adiabatic flames, the dormant stage can be quite long, as seen in Figure 6.

It is very interesting to encounter a flame that, after the initial external ignition source deposition, undergoes a sudden burst of reactivity and then appears extinguished before suddenly propagating again. Experimentally, one may mistakenly group the initial burst of high reactivity with that from the external ignition source and then attribute the apparent dormant stage as part of the entire ignition delay due to chemical effects. In actuality, the behavior is due to the stretch-promoted hibernation mode of pulsation. The duration of this extended hibernation is very sensitive to any loss/perturbation effects; in fact,

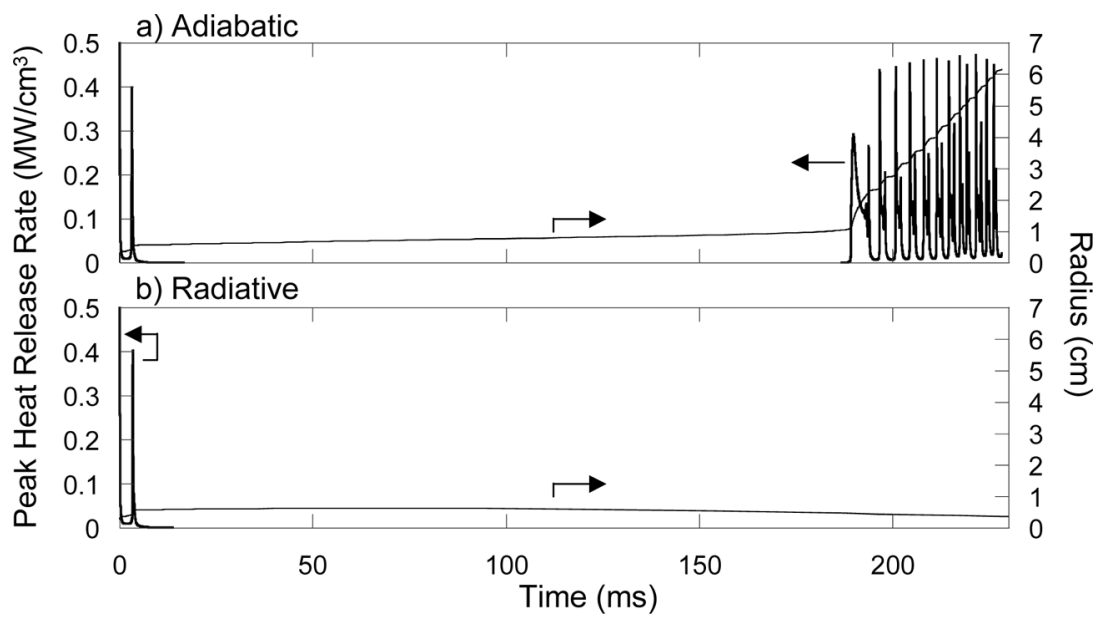

Figure 6. Outwardly, spherically propagating flame at $10 \mathrm{~atm}, \phi=5.0$. 
the duration was found to be noticeably different for simulations run on different machines due to different machine constants and, hence, numerical errors. Parenthetically, the simulation of the resumed, pulsatingpropagation portions is not machine dependent. Consequently, for the particular case of Figure 6, in contrast to that of Figure 5, radiative loss extinguishes the flame, akin to the pulsating extinction behavior observed in other works when radiative loss is considered for the hibernation mode. Figure 4 then presents the extreme case; where even with no heat loss, the flame cannot recover from the hibernation duration after the initial heat-release burst. This phenomenon is not seen in planar flames, which can always recover from long periods of substantially diminished reactivity with short intervals of high reactivity due to the infinite amount of thermal energy on the burned side of the doubly infinite domain, which can always diffuse forward to "reignite" the flame. Therefore, the adiabatic pulsating extinction in the spherical flame is conceptually analogous to the ignition limit where a propagating flame may not be dynamically realized from a localized initial structure of finite energy.

Since increasingly rich mixtures promote the hibernation mode, it is reasonable that this regime of pulsating extinction should neighbor the no-ignition boundary. Moreover, the regime appears as an island for which stretch can induce hibernation modes of characteristically weak bursts of heat release and large inactivity periods. Its top boundary coincides with the turning point of the no-ignition curve into larger equivalence ratios, where such weak pulsating modes are suppressed due to lowered activation energy from chemistry above the extended second limit. Its lower boundary is that of decaying pulsation, where stretch effects cannot engender the hibernation pulsation mode. Thus, ignition and extinction are intimately linked, where any consideration of propagating flame ignition for near-rich-limit flames should incorporate these combined stretch and thermal-diffusive effects. It is worth conjecturing that the flame radius at which continued pulsations resume after the hibernation period (see Figure 6) is related to the critical radius of $\mathrm{He}$ and Clavin (1993) needed for ignition of spherically propagating flames in near-rich-limit mixtures. More detailed study is needed. In summary, although the general finding is that stretch promotes the pulsating behavior in otherwise stable flames, it also promotes immediate extinction for sufficiently rich mixtures, whereby a propagating flame cannot be "ignited." 


\section{Radiative Effects on Flammability Boundaries}

For simplicity in presenting the role of radiative heat loss on the flame dynamics, the results given in this section are based on calculations employing optically thin radiation with the Planck mean absorption/ emission coefficients of Hubbard and Tien (1978), which also represents the most severe heat loss condition. With respect to the scope of this work, the effects of reabsorption have been found to be mainly quantitative (e.g., limited calculations revealed that reabsorption can shift the boundaries) and do not affect the discussion of the underlying physical mechanisms.

Figure 7 shows the stability, extinction, and non ignition boundaries of radiative, spherically-propagating, rich hydrogen/air flames established by local ignition from 1 to $20 \mathrm{~atm}$ at $298 \mathrm{~K}$ ambient. To compare with the adiabatic cases on an equal basis, deposition of ignition energy was done adiabatically, with radiative loss "turned" on after the ignition duration $\tau_{s}$. As can be seen, the boundaries are only moderately modified. Consistent with other work, radiative loss hardly affects the onset of instability due to the low ratio of heat loss to heat generation far

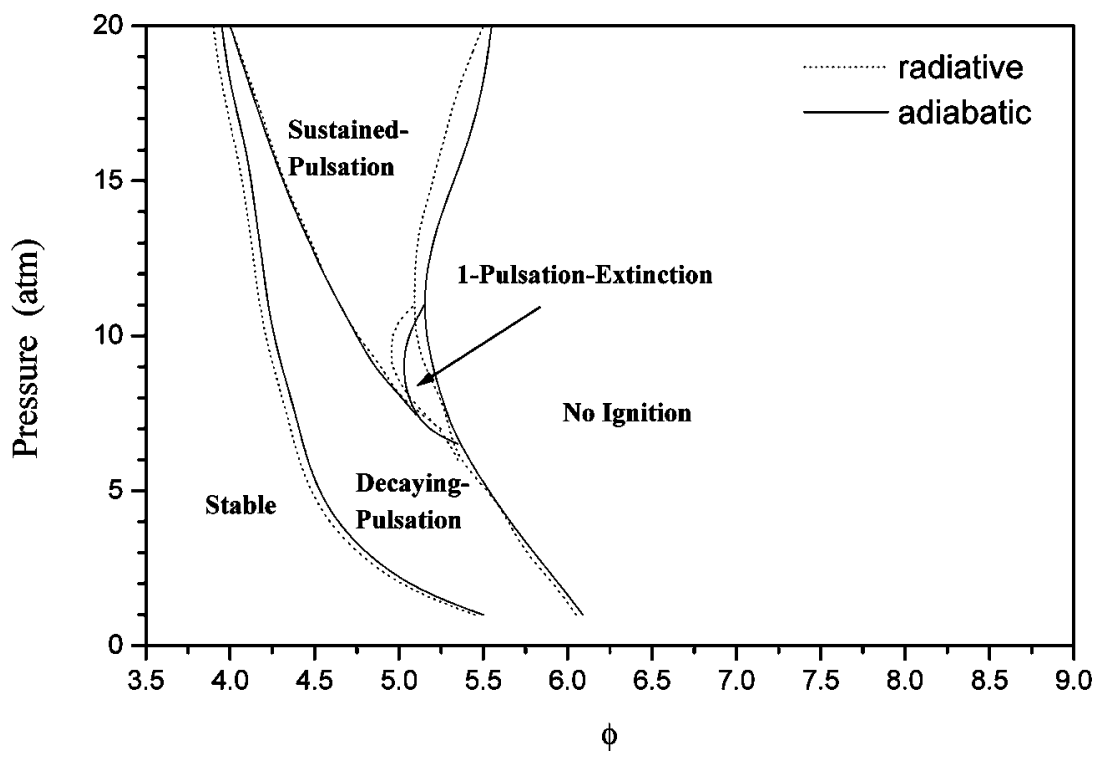

Figure 7. Stability and extinction boundaries of radiative rich $\mathrm{H}_{2}$ /air spherically propagating flames from 1 to $20 \mathrm{~atm}$ at $298 \mathrm{~K}$ ambient. 
from the flammability limit. Its strongest effect is on flames undergoing the weakly burning stage during the hibernating pulsating mode. For our centrally ignited flames, this corresponds to mixtures that exhibit the characteristics of Figure 6. As a result, radiative extinction encroaches into the sustained pulsation regime. Radiative loss also shifts most of the no-ignition boundary to the left. Specifically, for the limit bordering the island of adiabatic (one-burst) extinction, it extinguishes otherwise adiabatic weak single bursts. As a result, the island of 1-pulsation-extinction still exists (but is no longer purely adiabatic in nature) and is pushed to the left. Above $11 \mathrm{~atm}$, radiative loss effects, immediately after external energy deposition, extinguish reactivity so that otherwise adiabatic weak hibernation-mode bursts do not even appear, thereby causing the no-ignition curve to appear to shift into the regime of adiabatic sustained pulsation. A somewhat "hard" limit of ignition lies below $6 \mathrm{~atm}$, where pulsations are only stretchinduced, no sustained oscillations exist, and hibernation modes never appear. The effects of radiative loss on the thermal-diffusive instability resulting in extinction processes at the no-ignition boundary further emphasize the coupled nature of the pulsating instability and flame propagation for near-limit flame propagation ignition.

\section{Some Flame Structure Considerations}

Study of the flame structure evolution yields physical insight into the pulsating phenomenon. Returning to the flame response of Figure 5, we examine the flame structure at specific points in its propagation (Figure $8 a$ ). After external energy addition, the outwardly propagating flame undergoes an initial burst (A B C D E) of "high" reactivity representing the high-stretch-induced hibernating pulsation mode. Following the dormant period, the flame "re-ignites" and propagates in the perioddoubling mode (F G H I J) until finally propagating in the sustained monochromatic mode as mentioned earlier.

Figures $8 b$ and $8 c$ show elements of the flame structure (temperature, heat-release rate, $\mathrm{O}_{2}$ mole fraction, and $\mathrm{H}_{2}$ mole fraction) at different points in the trajectory and peak-heat-release history of the flame corresponding to Figure $8 a$. For points A through $\mathrm{E}$, the flame structure, in terms of the diffusive properties plotted, is that of the conventional propagating premixed flame with a localized structure, characterized by exponential decay in the profiles. As can be seen, excess enthalpy 


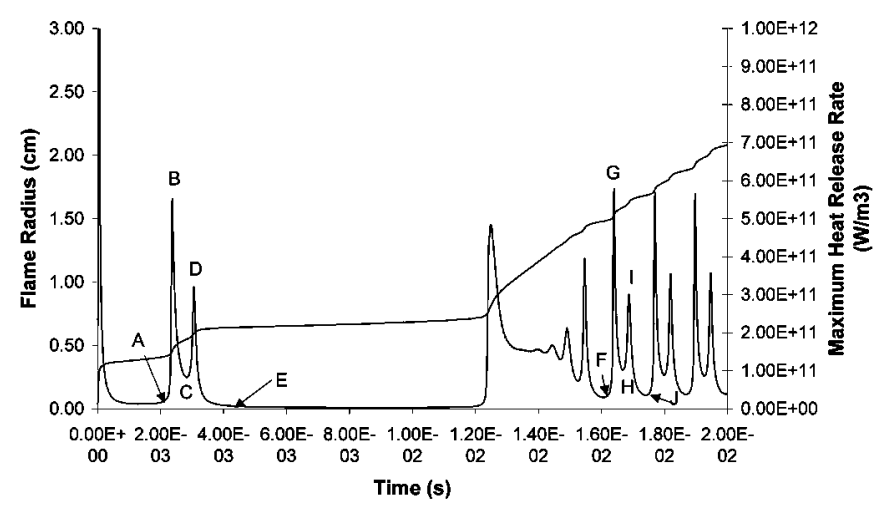

(a) Flame history
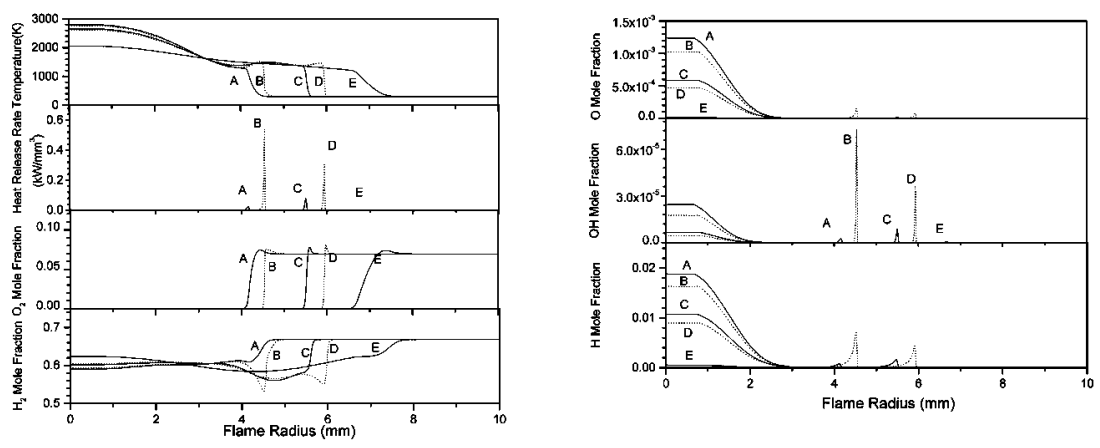

(b) Flame structure of initial burst
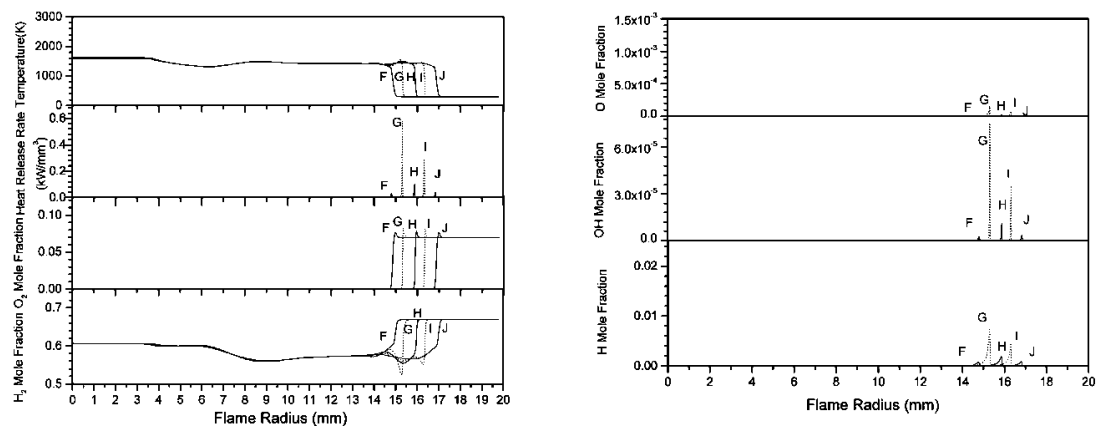

(c) Flame structure of subsequent oscillation

Figure 8. Flame structure evolution for case of Figure 5. 
from ignition is present, supporting the flame, so that even by point $\mathrm{E}$, where there is negligible heat release, there is still plenty of thermal energy in the burned gas to support hibernation and allow the flame to revive. With oxygen as the deficient species, complete consumption of it occurs through the heat-release zone. Varying amounts of hydrogen leak through the heat-release zone at different points corresponding to the peak-heat-release history, with significantly different instantaneous profiles of hydrogen mole fraction.

In Figure $8 c$, the flame undergoes a single oscillation in the perioddoubled mode. As can be seen, the overall gas-phase response is slow compared to that induced by the local oscillations. As a result, the overall flame response to stretch is not what one would calculate instantaneously, but rather an "average" flame stretch that is monotonically decreasing.

\section{Elevated Ambient Temperature Effects}

We now study the competing aspects of increased global enthalpy and stretch effects on the pulsating instability. Figure 9 shows the stability, extinction, and nonignition boundaries of adiabatic, spherically propagating, rich hydrogen/air flames established by local ignition from 1 to $20 \mathrm{~atm}$ at $400 \mathrm{~K}$ ambient. As can be seen, the entire boundary map, compared to that for $298 \mathrm{~K}$ ambient, is significantly shifted to the right. Increased enthalpy moderates stretch-induced pulsations, shifting the onset boundary to the right. Even more so, the ignition limit is shifted to richer mixtures, with elevated ambient temperature facilitating flame propagation establishment. Interestingly, the second stable regime at high pressures of Figure $1 a$ is not revealed. This effect is likely due to increased ambient enthalpy, which reduces $Z e$, shifting sustained pulsation modes to richer mixtures and thereby pushing the entire map of Figure $1 a$ to higher equivalence ratios-thus also affecting the left boundary of sustained pulsations of Figure 9. Such an explanation would support why sustained pulsations are still not seen for pressures below about $8 \mathrm{~atm}$. The 1-pulsation-extinction regime still exists but becomes squeezed and moved somewhat toward lower pressures. Nonetheless, ignition constraints still limit the ranges for which the pulsating phenomenon can be observed experimentally.

The varying responses of adiabatic flames for a fixed equivalence ratio and pressure for different ambient temperatures of 298,400 , and 


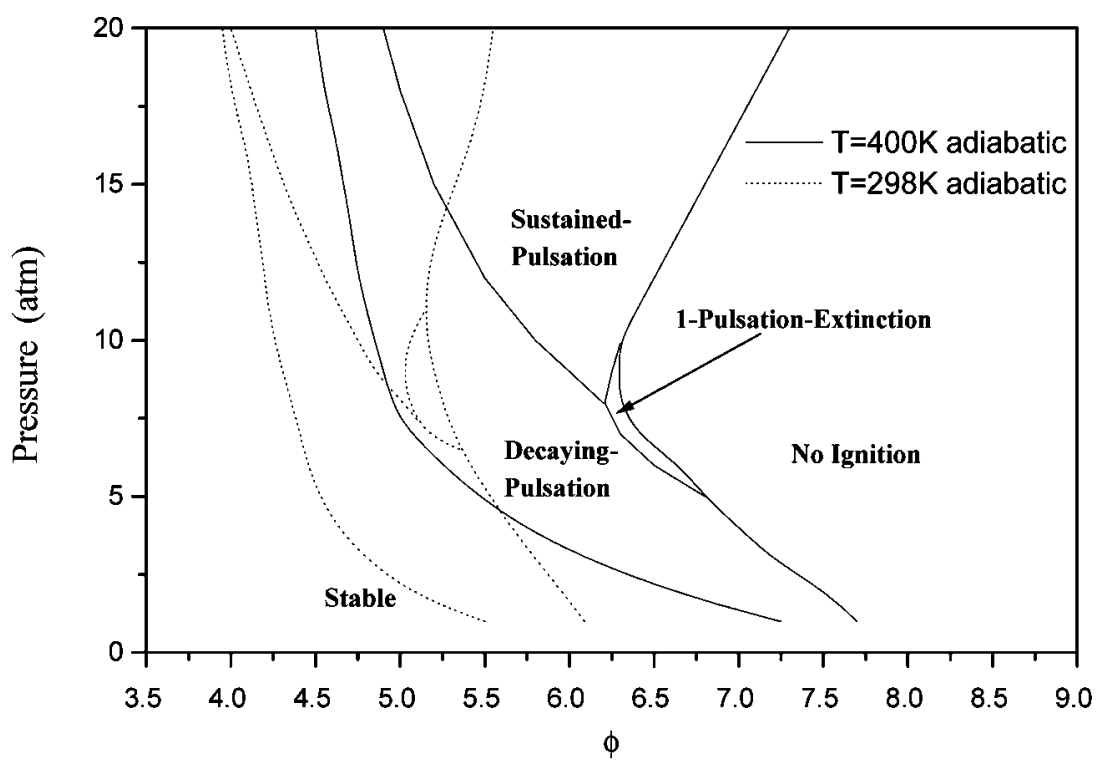

Figure 9. Stability and extinction boundaries of adiabatic, rich $\mathrm{H}_{2}$ /air spherically propagating flames from 1 to $20 \mathrm{~atm}$ at $400 \mathrm{~K}$ ambient.

$500 \mathrm{~K}$ (below that which would induce chemical reactions at the upstream boundary) were examined. At $298 \mathrm{~K}$, a propagating flame is nonignitable, where any movement of the heat-release front is supported by the external ignition source. By increasing the ambient temperature, ignition is facilitated so that, at $400 \mathrm{~K}$, a propagating sustained pulsating flame can be established. However, by $500 \mathrm{~K}$, elevated global enthalpy has reduced $\mathrm{Ze}$ so much below that required for Sivashinsky's criterion for the transition boundary that not even high stretch effects can induce pulsation, and a stable spherically propagating flame is maintained.

\section{CONCLUDING REMARKS}

The study has shown that the region of instability in the pressure versus equivalence ratio domain for outwardly propagating flames is significantly narrowed, even for the adiabatic case, when compared with that for planar flames, due to the presence of the local ignition limit despite stretch-induced pulsation modifying the "onset" boundary. An adiabatic mode of extinction, due to high stretch at small flame radii, is the result 
of the hibernation mode appearing first during flame propagation. Radiative loss in outwardly propagating spherical flames further reduces the region of instability due to transient extinction. From flame structure investigations, the overall gas-phase response appears to be slow compared with that induced locally by the pulsating behavior. Thus, the overall flame response to stretch is not that which would be calculated instantaneously, but rather an "average" flame stretch that is monotonically decreasing in our outwardly propagating flame. Moreover, elevated temperatures shift the entire flammability map to the right because, although ignition of propagating flames is promoted for richer mixtures, additional enthalpy facilitates overcoming of the activation energy (reducing $Z e$ ), thereby stabilizing the flame. Nonetheless, a major finding of the work is the coupled nature of local ignition and extinction from the combined effects of stretch and thermal-diffusive instability for rich-limit $(L e>1)$ flames. Any consideration of flame propagation ignition must address the competition between excess enthalpy from external energy deposition and stretch/heat-loss-affected pulsation instability.

\section{REFERENCES}

Christiansen, E.W., Sung, C.J., and Law, C.K. (1998) Pulsating instability in near-limit propagation of rich hydrogen/air flames. Proc. Combust. Instit., 27, 555-562.

Christiansen, E.W., Law, C.K., and Sung, C.J. (2001) Steady and pulsating and extinction of rich hydrogen/air flames at elevated pressures. Combust. Flame, 124, 35-49.

Farmer, J.R. and Ronney, P.D. (1990) A numerical study of unsteady nonadiabatic flames. Combust. Sci. Technol., 73, 555-574.

Goyal, G., Maas, U., and Warnatz, J. (1995) Simulation of the behavior of rich hydrogen-air flames near the flammability limit. Combust. Sci. Technol., 105, 183-193.

He, L. and Clavin, P. (1993) Premixed hydrogen-oxygen flames. Part II: Quasiisobaric ignition near the flammability limits. Combust. Flame, 93, 408-420.

Hubbard, G.L. and Tien, C.L. (1978) Infrared mean absorption coefficients of luminous flames and smoke. J. Heat Transf., 100, 235-239.

Joulin, G. and Clavin, P. (1979) Linear stability analysis of nonadiabatic flames: Diffusional-thermal model. Combust. Flame, 35, 139-153.

Kailasanath, K., Ganguly, K., and Patnaik, G. (1993) Dynamics of flames near the rich-flammability limit of hydrogen-air mixtures. Progress in Astronautics and Aeronautics, AIAA, Washington, DC, Vol. 151, pp. 247-262. 
Law, C.K. and Egolfopoulos, F.N. (1992) A unified chain-thermal theory of fundamental flammability limits. Proc. Combust. Instit., 24, 137-144.

Mueller, M.A., Kim, T.J., Yetter, R.A., and Dryer, F.L. (1999) Flow reactor studies and kinetic modeling of the $\mathrm{H}_{2} / \mathrm{O}_{2}$ reaction. Int. J. Chem. Kinet., 31, 113-125.

Rogg, B. (1982) The effect of Lewis number greater than unity on an unsteady propagating flame with one-step chemistry. In Peters, N. and Warnatz, J. (Eds.) Numerical Methods in Laminar Flame Propagation, Friedr, Vieweg \& Sohn, Brunswick, Germany, pp. 38-48.

Rogg, B. and Wang, W. (1995) RUN-1DL: The Cambridge Universal Flamelet Computer Code, User Manual, University of Cambridge, Cambridge, England.

Sivashinsky, G.I. (1974) On a converging spherical flame front. Int. J. Heat Mass Transf., 17, 1499-1506.

Sivashinsky, G.I. (1977) Diffusional-thermal theory of cellular flames. Combust. Sci. Technol., 15, 137-146.

Spalding, D.B. (1957) A theory of inflammability limits and flame-quenching. Proc. R. Soc. Lond. A, 240, 83-100.

Sung, C.J., Makino, A., and Law, C.K. (2002) On stretch-affected pulsating instability in rich hydrogen/air flames: Asymptotic analysis and computation. Combust. Flame, 128, 422-434.

Tse, S.D., Zhu, D.L., and Law, C.K. (2000) Morphology and burning rates of expanding spherical flames in $\mathrm{H}_{2} / \mathrm{O}_{2} /$ Inert mixtures up to 60 atmospheres. Proc. Combust. Instit., 28, 1793-1800.

Williams, F.A. (1985) Combustion Theory, Benjamin-Cummings, Palo Alto, CA. 